\title{
Faux anévrisme de l'artère brachiale chez un utilisateur de drogues injectables
}

\author{
Adam Kovacs-Litman MD, Wayne L. Gold MD
}

- Citation : CMAJ 2021 March 29;193:E448. doi : 10.1503/cmaj.201843-f

Voir la version anglaise de l'article ici : www.cmaj.ca/lookup/doi/10.1503/cmaj.201843

$\mathbf{U}$

n homme de 39 ans consommant du fentanyl injectable est venu consulter à l'urgence pour une masse pulsatile douloureuse à croissance progressive présente dans la fosse cubitale droite depuis 1 mois (figure 1 ). Il a dit ne pas avoir fait de fièvre. À l'auscultation, un bruit dans la masse était évocateur d'un anévrisme. Nous avons demandé une angiotomodensitométrie et une échographie Doppler du bras droit. Les résultats concordaient avec un faux anévrisme de l'artère brachiale, où s'étaient logés des fragments d'aiguille (annexe 1, accessible en anglais au www.cmaj.ca/ lookup/doi/10.1503/cmaj.201843/tab-related-content). Nous avons posé un diagnostic provisoire de faux anévrisme infecté (mycotique).

Après 3 hémocultures sans croissance de micro-organismes, nous avons prescrit une antibiothérapie empirique à la vancomycine et à la pipéracilline-tazobactam, médicaments que nous avons remplacés quelques jours plus tard par une association d'amoxicilline et d'acide clavulanique $(875 \mathrm{mg} / 125 \mathrm{mg}$ par voie orale aux $12 \mathrm{~h}$ ) pour un total de 4 semaines. Le faux anévrisme a fait l'objet d'un pontage. Les tissus potentiellement infectés et les fragments d'aiguille ont été laissés in situ étant donné l'important risque opératoire lié à leur extraction. Le patient s'est rétabli sans complications.

Le terme faux anévrisme décrit une atteinte structurelle aux 3 couches de la paroi artérielle menant à la formation d'une poche de sang; il découle habituellement d'un trauma, par exemple l'injection de drogues ${ }^{1}$. Dans ce contexte, l'infection peut survenir par inoculation directe de microorganismes dans la paroi vasculaire ou par propagation des micro-organismes d'un faux anévrisme existant dans la circulation systémique ${ }^{2}$. En général, les faux anévrismes mycotiques prennent la forme de masses pulsatiles douloureuses au volume croissant et sont accompagnés de signes d'infection locale. Dans une série de cas sur 50 patients ayant un anévrisme mycotique attribuable à l'injection de drogues, on a recensé les symptômes suivants : fièvre $(48 \%)$, masse indurée sensible $(92 \%)$, masse pulsatile $(52 \%)$ et présence d'un bruit $(48 \%)^{2}$. Les résultats des hémocultures étaient positifs dans une proportion de $50 \%-85 \%^{3}$.

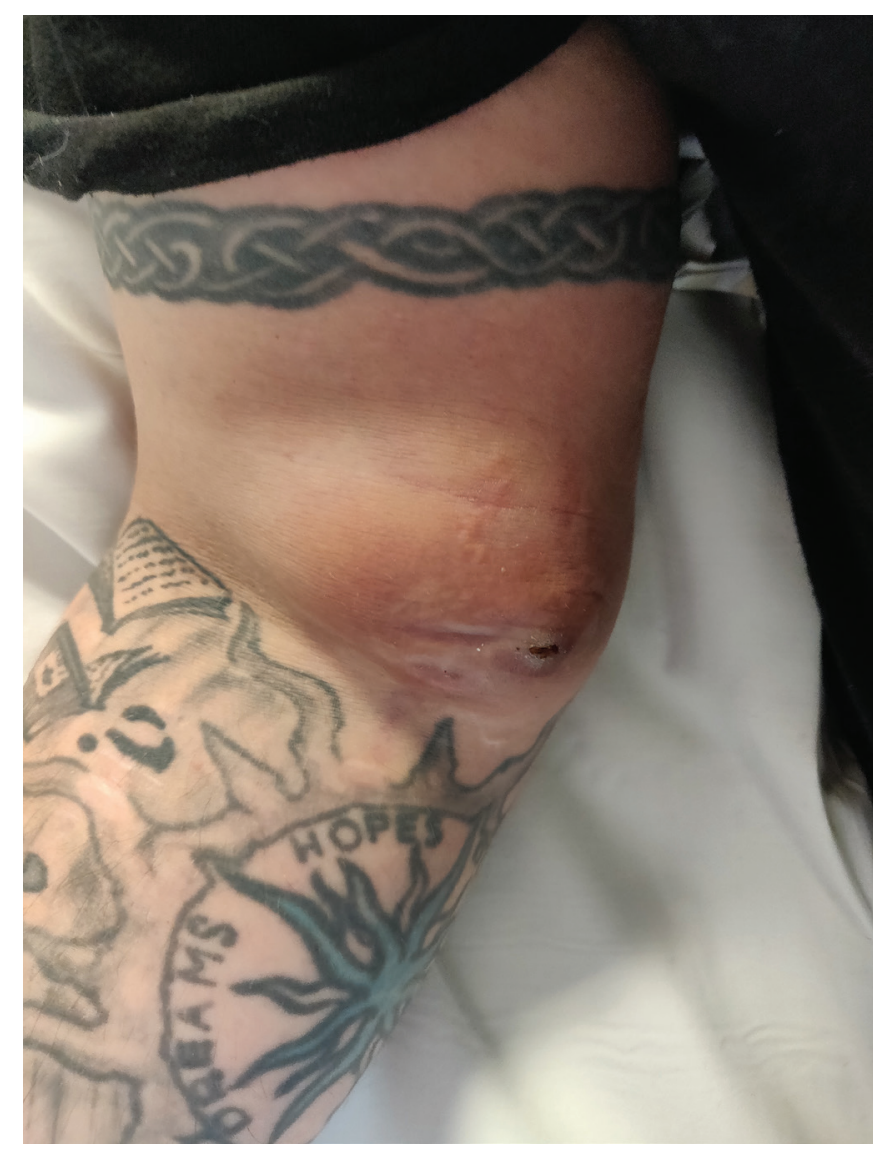

Figure 1 : Bras droit d'un homme de 39 ans consommant des drogues injectables; on y voit une masse pulsatile (diamètre de $8 \mathrm{~cm}$ ) au-dessus de la fosse cubitale, de même qu'un site de ponction et un érythème sus-jacent.

La prise en charge comprend une antibiothérapie, une intervention chirurgicale - une excision si possible - et une revascularisation, au besoin ${ }^{2}$. Il importe de distinguer le faux anévrisme mycotique - surtout s'il est non pulsatile d'un abcès, puisque toute tentative d'incision et de drainage d'un faux anévrisme risque de provoquer une hémorragie catastrophique. 


\section{Références}

1. Regus S, Lang W. Rupture risk and etiology of visceral artery aneurysms and pseudoaneurysms: a single-center experience. Vasc Endovascular Surg 2016;50:10-5.

2. Johnson JR, Ledgerwood AM, Lucas CE. Mycotic aneurysm. New concepts in therapy. Arch Surg 1983;118:577-82.

3. Maeda H, Umezawa H, Goshima M, et al. Primary infected abdominal aortic aneurysm: surgical procedures, early mortality rates, and a survey of the prevalence of infectious organisms over a 30-year period. Surg Today 2011; 41:346-51.

Les images cliniques sont choisies pour leur caractère particulièrement intéressant, classique ou impressionnant. Toute soumission d'image de haute résolution claire et bien identifiée doit être accompagnée d'une légende aux fins de publication. On demande aussi une brève explication (300 mots maximum) de la portée éducative des images, et des références minimales. Le consentement écrit du patient au regard de la publication doit être obtenu avant la soumission.
Intérêts concurrents : Aucun déclaré.

Cet article a été révisé par des pairs.

Les auteurs ont obtenu le consentement du patient.

Affiliations : Département de médecine (Gold, Kovacs-Litman), Université de Toronto; Divisions de médecine interne générale et d'infectiologie (Gold), Réseau universitaire de santé, Toronto, Ont.

Propriété intellectuelle du contenu : Il s'agit d'un article en libre accès distribué conformément aux modalités de la licence Creative Commons Attribution (CC BY-NC-ND 4.0), qui permet l'utilisation, la diffusion et la reproduction sur tout médium à la condition que la publication originale soit adéquatement citée, que l'utilisation se fasse à des fins non commerciales (c.-à-d., recherche ou éducation) et qu'aucune modification ni adaptation n'y soit apportée. Voir : https://creativecommons.org/licenses/by-nc-nd/4.0/deed.fr.

Correspondance : Adam Kovacs-Litman, adam.kovacslitman@mail.utoronto.ca 